\title{
ASESORÍAS INNOVADORAS EN RECURSOS HUMANOS PARA LAS PYMES MEDIANTE EL USO DE LA RED DE INTERNET
}

\author{
NYDIA ISABEL VEGA ARTAVIA \\ Escuela de Ciencias de la Administración \\ Universidad Estatal a distancia \\ nvegar07@gmail.com
}

\section{RESUMEN}

Este estudio examina la viabilidad de la implementación de una empresa de consultoría en recursos humanos que ofrezca sus servicios por medio del aprovechamiento de las TIC que ofrece Internet. La necesidad de este tipo de servicio existe y ha quedado comprobada en Costa Rica en diversos estudios realizados por profesionales en recursos humanos, las Mipymes requieren los servicios de asesoría en recursos humanos para mejorar la gestión mediante la identificación de los problemas y la implementación de soluciones propuestas por asesores externos. Los profesionales que brindan servicios de asesoría deben ser personas que tengan experiencia en la materia y formación académica en el área de recursos humanos, además de habilidades tecnológicas que contribuyan a dar un servicio de calidad a los pequeños y medianos empresarios.

PALABRAS CLAVE: CONSULTORÍAS, EMPRENDIMIENTOS, COMPETENCIAS, TECNOLOGÍAS DE LA INFORMACIÓN.

\section{ABSTRACT}

This study examines the feasibility of implementing an enterprise for human resources consultancy offering services through the use of the ICT that Internet offers. The need for this type of services exists and has been proven in Costa Rica in various studies by human resource professionals. MSMEs require consulting services in human resources to improve management by identifying problems and implementing solutions proposed by external advisors. The profes- sionals who provide counseling services should be people with experience in the field and academic training in the area of human resources, plus technology skills that could guarantee quality services to small and medium entrepreneurs.

KEYWORDS: CONSULTING, ENTREPRENEURSHIP, SKILLS, INFORMATION TECHNOLOGY.

\section{INTRODUCCIÓN}

Este artículo presenta un análisis acerca de la necesidad por parte de las Pymes (pequeñas y medianas empresas) de capacitación en recursos humanos, y la importancia de que las empresas consultoras les ofrezcan a los empresarios soluciones adecuadas a sus necesidades, a precios que ellos puedan solventar con su presupuesto.

A partir de esta investigación se pretende dar respuesta a la siguiente pregunta:

¿Por qué insertar en el mercado un servicio de consultorías en recursos humanos para las Pymes mediante el uso de la red?

En la actualidad las empresas compiten en dos mundos: el mundo real de recursos tangibles y el mundo virtual, por lo que cada vez son más las empresas que han hecho su entrada en el ciberespacio. 
El uso de Internet en la actualidad por parte de las empresas y su aprovechamiento como medio de comunicación es vital, difícilmente las Pymes pueden sobrevivir sin ingresar a la red, por lo que se ven forzadas a diseñar estrategias de sistemas de información (SI), la red es una nueva fuente de oportunidades y de amenazas para las empresas, sea cual sea su sector de actividad e incluso su tamaño.

El protocolo TPC/IP (transmission Control Protocol/ Internet Protocol) ha hecho posible que diferentes sistemas operativos y computadoras se conecten entre sí y se ha consolidado como el principal lenguaje de comunicación en red (González. 2001) Las herramientas de navegación más conocidas son el correo electrónico (e- mail), las listas de correo automatizadas, el robot de correo, los servidores de información, telnet, los servidores de área amplia, constituyéndose como la herramienta más popular el conjunto de servicios World-Wide Web (WWW) en todos los ámbitos, especialmente en el empresarial.

Las empresas obtienen de la red diversos beneficios, no solo la utilidad comercial, pueden localizar información precisa, acceder a bases de datos y comunicarse con usuarios situados en cualquier lugar del mundo.

La Internet tiene características que la convierten en un medio de comunicación sin comparación en el mundo empresarial, por razones como su carácter público, el alcance global, la capacidad de conexión, la propiedad compartida, la flexibilidad y diversidad de plataformas, además de las ventajas de costo (González, 2001).

La introducción de las tecnologías de información y comunicación(TIC), modificó la forma de producción de bienes y servicios, los cuales se preparan y ofrecen según las especificaciones de los clientes, la globalización por su parte a contribuido a identificar nichos de mercado en otras zonas geográficas, es decir, las empresas hoy día pueden estar distribuidas geográficamente, ter- ciarizar servicios (outsourcing - offshoring), incorporarse a redes de empresas, reclutar trabajadores a distancia; no obstante el marketing, la publicidad y el posicionamiento en buscadores, entre otras acciones, requieren de nuevos lenguajes y técnicas, de ahí la necesidad de que los empresarios adquieran nuevas habilidades, con el objetivo de contar con las condiciones necesarias para realizar las transacciones (compras, ventas, reservas, publicidad, etcétera) de comercio electrónico mediante redes telemáticas.

\section{MARCO TEÓRICO}

El concepto "emprendedor" tiene su origen en una antigua palabra francesa entrepreneur, utilizada en la Edad Media para denominar a los comerciantes que asumían el riesgo de viajar a otras ciudades para realizar intercambios comerciales, con la particularidad de ser financiados por un tercero., un emprendedor es una persona que asume riesgo del éxito o el fracaso de la actividad comercial que desarrolla, "el emprendedor es entonces quien descubre, evalúa y explota oportunidades rentables, tomando en cuenta el riesgo, alerta a las oportunidades y necesidad por la innovación" (Hisrich, Peters y Shepherd, 2005). Por otra parte, el concepto de empresa se refiere a "emprender una actividad que implica trabajo o que presenta dificultades" (Roberts y Woods, 2005, p.46), es decir un empresario es un emprendedor.

Asimismo, un empresario (businessman, entrepeneur) es capaz de arriesgar esfuerzo, tiempo y recursos para poner en marcha y desarrollar una unidad de producción y/o prestación de servicios para la satisfacción de determinadas necesidades y/o deseos existentes en la sociedad a cambio de una utilidad o beneficio (Thompson, 2010). En términos simples, es quien crea o adquiere un negocio o empresa, y luego se dedica a trabajar en él y hacerlo crecer, además de cumplir con el siguiente proceso (Tompson, 2010): 
1. Identifica una oportunidad: En primer lugar un empresario posee la suficiente visión como para reconocer una oportunidad de negocios.

2. Asume riesgos: Una vez identificada la oportunidad de negocios, se informa, investiga y la analiza; si considera que el negocio en ciernes podría ser rentable, toma los riesgos necesarios y asume la responsabilidad de su decisión.

3. Reúne recursos: Después de decidir iniciar su negocio, reúne rápidamente los recursos necesarios (financieros, tecnológicos, humanos, entre otros) que le permiten poner en marcha su empresa.

4. Innova: utiliza su creatividad para diseñar un producto único que ofrezca una diferenciación con respecto a los de sus competidores y satisfaga necesidades de los consumidores.

5. Desarrolla procesos: Posteriormente, crea procesos o sistemas que hagan funcionar eficientemente su negocio. Procesos o sistemas sobre cómo adquirirá los insumos, como producirá sus productos, cómo los distribuirá al público, como los promocionará, etcétera.

6. Lidera: Una vez que ha iniciado su negocio, ejerce su liderazgo para inducir y motivar a sus trabajadores a que alcancen los objetivos de su empresa.

7. Contribuye a la comunidad: Por último, contribuye con la comunidad, ya sea brindando un bien o un servicio útil a los consumidores, incentivando la economía, generando empleo y creando nuevos puestos de trabajo.

La empresa es la célula creadora de riqueza dentro de la sociedad, riqueza que permite el desarrollo económico, el acceso a la cultura, a la salud, la seguridad y todos los elementos que proporcionan bienestar y calidad de vida Kantis (2004); forma así parte de la contribución que el empresario hace a la comunidad.

Con el objetivo ampliar el concepto de empresa a continuación, en la tabla 1 se muestra un resumen acerca de los aportes de algunos teóricos a este respecto, definiciones tomadas del estudio acerca del desarrollo emprendedor, Kantis (2004).

\section{TABLA 1}

\section{CONCEPTO DE EMPRESA}

\begin{tabular}{|c|c|}
\hline Autor & \multicolumn{1}{|c|}{ Definición } \\
\hline $\begin{array}{c}\text { McClelland, } \\
(1961)\end{array}$ & $\begin{array}{l}\text { Por su necesidad de logros, el deseo de ser } \\
\text { independiente, la capacidad de tolerar la } \\
\text { ambigüedad y el riesgo, la perseverancia y la } \\
\text { autoconfianza. }\end{array}$ \\
\hline $\begin{array}{c}\text { Gilder, } \\
\text { (1984) }\end{array}$ & $\begin{array}{l}\text { Por su capacidad de aprender, lo cual le } \\
\text { permite a pesar de los fracasos y condiciones } \\
\text { adversas, lograr sus objetivos. }\end{array}$ \\
\hline $\begin{array}{c}\text { Schumpeter, } \\
\text { (1934) }\end{array}$ & Resalta el rol innovador. \\
\hline $\begin{array}{c}\text { Baumol, } \\
\text { (1997) }\end{array}$ & $\begin{array}{l}\text { Es un imitador que explota una oportunidad } \\
\text { de negocios identificada por otra persona. }\end{array}$ \\
\hline $\begin{array}{c}\text { Brockhaus, } \\
\text { (1980) }\end{array}$ & $\begin{array}{l}\text { Asocia la actividad empresarial a la propiedad } \\
\text { del negocio y a la toma de riesgos. }\end{array}$ \\
\hline $\begin{array}{c}\text { Jennings, } \\
\text { (1994) }\end{array}$ & Visión operativa, quien funda una empresa. \\
\hline
\end{tabular}

Fuente: Elaboración propia.

Sea que se le denomine empresario o emprendedor, estas personas presentan características y desempeñan funciones que los diferencian de los demás y no existe una respuesta a la pregunta de si los emprendedores nacen o se hacen, sin embargo, existen individuos que poseen la habilidad para crear empleo para sí mismos y para otros, como un medio para el generar ingresos contribuyendo al desarrollo económico de un país. 
En Costa Rica las pequeñas y medianas empresas (Pymes), pertenecen al sector privado, que es el sector de la economía que busca lucrar con su actividad y no está controlado por el Estado. La mayoría de estas se encuentran en el sector del comercio y servicios, de acuerdo con datos de la CCSS del año 2009 representan casi $98 \%$ de las empresas del país.

Según su dimensión las empresas en Costa Rica se clasifican en:

- Micro empresa: de 1 a 5 trabajadores.

- Pequeña empresa: de 6 a 30 trabajadores.

- Mediana empresa: de 31 a 100 trabajadores.

- Gran empresa: más de 100 trabajadores.

Las Pymes representan un importante sector en la economía del país, acorde con estimaciones del Ministerio de Economía, Industria y Comercio, el conglomerado de micro, pequeñas y medianas empresas (Mypimes) constituye aproximadamente $98 \%$ del total de empresas privadas del país, concentradas en los sectores de comercio y servicios, lo cual concuerda con los datos de la CCSS. Las Mipymes en total generan 48,4\% del empleo en el sector privado. (Datos publicados por el Observatorio Mipymes, 2008)

Según Aragón y Rubio (2006) Las Pymes son el motor económico principal de la expansión económica y del empleo en la mayoría de los países. En un estudio empírico realizado por los investigadores, en el cual analizaron 431 empresas, identificaron los siguientes rasgos que caracterizan a las Pymes más competitivas:

1. Ocupan una posición tecnológica, fuerte, la adquisición y uso que hacen de la tecnología las posiciona por delante de la competencia.

2. Recaban información sobre el negocio, muestran buena imagen ante el mercado y se anticipan con rapidez a los cambios.
3. Realizan procesos de selección de personal rigurosos que permiten la entrada de personal que se adapta a las necesidades de la empresa como un todo.

4. Fomentan el trabajo en equipo y la participación de los trabajadores.

5. Sus dirigentes se caracterizan por ser líderes naturales.

6. Se ocupan por obtener información actualizada de los mercados financieros.

7. Creen en el compromiso con los valores, el cumplimiento de la misión y los objetivos de la empresa.

Las Pymes en Costa Rica emplean aproximadamente la tercera parte del mercado laboral del país, lo que demuestra su grado de importancia en la economía nacional, satisfacen necesidades que demanda el mercado en todos los sectores de la economía, constituyéndose en una importante fuente de empleo, por lo que deberían de contar con procesos de selección de personal rigurosos, adaptados a sus necesidades y fomentar el trabajo en equipo de sus integrantes, al igual que las grandes empresas estás van a requerir asesoría empresarial en áreas como contabilidad, finanzas, mercadeo y recursos humanos, con el objetivo de mejorar su desempeño empresarial, servicio que es prestado por empresas consultoras en administración.

Las empresas de consultoría contribuyen a resolver situaciones internas, por medio de soluciones propuestas por personas externas (asesores) surge así la necesidad de contratar sus servicios.

Otra característica que tienen las Mipymes es que cuentan con un presupuesto reducido para invertir en consultorías, y en la mayoría de los casos carecen de alguien dedicado solamente a 
las funciones de recursos humanos, por tanto es una de las áreas en las cuales requieren asesoría de un profesional.

Una consultoría es un servicio que presta un profesional calificado, con capacidad, conocimiento y experiencia, cuya tarea consiste en la identificación e investigación de problemas relacionados con la gestión empresarial para así contribuir a la solución de problemas y el logro de objetivos (Zapata, Rivillas y Cardona, 2010).

De la misma forma, la consultoría es también un servicio al cual empresarios, directores y gerentes de empresas pueden recurrir en aquellos casos que detectan la necesidad de ayuda en la solución de problemas. Es un servicio independiente, que presta un profesional (consultor) que debe estar en condiciones de hacer su propia evaluación de cualquier situación, decir la verdad y recomendar con franqueza y objetividad las medidas que ha de adoptar la organización.

Según Gómez y Múnera (1998), en un proceso de consultoría pueden identificarse tres etapas genéricas que son el diagnóstico, la intervención y el seguimiento. En la primera etapa se realiza un análisis de la situación, los síntomas, las causas y condiciones críticas implicadas, a partir de este estudio se presenta una propuesta de intervención que requiere de consenso entre el cliente y el asesor; durante la segunda etapa se implementa la propuesta aprobada; finalmente, en la tercera etapa se evalúan los resultados obtenidos, el consultor acompaña al cliente para que pueda implementar los ajustes requeridos (Zapata et ál., 2010).

El proceso de consultoría es una actividad conjunta del consultor y el cliente, destinada a resolver un problema concreto y a aplicar los cambios deseados en la organización.

El enfoque de las relaciones humanas de la administración puso de manifiesto la importancia de gestión de los recursos humanos, es a finales de la década de 1950 que proliferan empresas consultoras que ofrecen sus servicios en áreas como desarrollo organizacional, rediseño de trabajo y administración de personal (Barley y Kunda, 1992). En las siguientes décadas el servicio que prestan se ha ido diversificando; según Zapata et ál. (2010), en la actualidad la principal línea de negocio es la consultoría en gerencia de operaciones con un 36,30\% del mercado, seguida por la línea de tecnologías de información $(22,20 \%)$ recursos humanos (14,20\%), estrategia corporativa $(14,00 \%)$ y servicios de outsourcing (13,30\%).

Existen diversas formas de clasificar las consultorías, dependiendo del vínculo existente entre el consultor y la empresa, así puede clasificarse en interna o externa; externa cuando el consultor es totalmente independiente del cliente desde el punto de vista jurídico y administrativo e interna cuando el consultor forma parte de la empresa en la cual presta sus servicios (Zapata, et ál., 2010).

También pueden clasificarse según el proceso involucrado, las hay en gestión estratégica, gestión financiera, mercadeo, producción, calidad, productividad, en sistemas de información, y en gestión de recursos humanos (Kubr, 2002), siendo esta última el objeto central de esta investigación.

La gestión humana se entiende tradicionalmente como la función, el departamento, las prácticas y la profesión relacionada con las operaciones de reclutamiento, selección, entrenamiento, remuneración, beneficios, comunicación, higiene y seguridad laboral en las organizaciones (Chiavenato, 2007).

En un sentido más amplio la administración de recursos humanos es la encargada de establecer las prácticas y políticas en materia de personal en las empresas, es el departamento que se encarga de atraer, seleccionar, integrar, capacitar, evaluar y mantener el recurso humano que la 
empresa requiere para el logro de los objetivos y metas, por tanto Ulrich (1997) plantea la gestión de los recursos humanos como un proceso estratégico.

Las consultorías en recursos humanos pueden ayudar a definir el perfil estratégico del área: orientado a la innovación, al reforzamiento o a los costos (Schuler y Jackson, 1987); además sirven de apoyo para revisar, orientar e implementar los procesos según la estrategia adoptada.

Actualmente, en Costa Rica las relaciones humanas dentro de las organizaciones son complejas, diversificadas y difíciles de manejar, algunas condiciones de empleo como el trabajo y la remuneración están reguladas por la legislación costarricense, por consiguiente en asuntos de personal el consultor debe estar plenamente consciente del marco jurídico y de las relaciones de trabajo existentes.

Se considera al personal como el recurso más valioso de una organización (Chiavenato, 2011) y de esa premisa se extraen varias conclusiones con respecto a la forma de tratar a los trabajadores y de motivarlos para que mejoren su rendimiento, la función de la dirección, la inversión en actividades de formación y desarrollo o la elección de sistemas de perfeccionamiento del personal.

Algunos de los motivos claves, de acuerdo con los hallazgos obtenidos en un estudio realizado por Zapata et ál. (2010), para la contratación de una consultoría son el interés por la formación del personal $(47,27 \%)$, los requerimientos legales $(47,27 \%)$, el interés por mejorar el clima organizacional $(43,64 \%)$, el requerimientos del plan estratégico (40,00\%), el deseo de tener una ventaja competitiva $(38,18 \%)$ y el deseo de incrementar la productividad (32,73\%).

Los profesionales que brindan asesoría a las empresas en materia de recursos humanos deben mostrar competencias como la capacidad para diagnosticar problemas, así como para evaluar situaciones, debe también conocer la gestión de recursos humanos, las condiciones del empleo, el trabajo, la remuneración, la legislación vigente (normativa), el papel de los sindicatos, reconocer la importancia de la motivación de los empleados, la importancia de evaluación del desempeño y formación y desarrollo de personal, y la vinculación de la cultura y los valores. Es decir, lo anterior es lo que las empresas de consultoría en recursos humanos deben a ofrecer a sus clientes.

Los servicios que ofrece un negocio de consultoría en gestión de recursos humanos son susceptibles a dividirse en ramas específicas, de acuerdo con las necesidades del cliente:

Los recursos humanos son un elemento fundamental para el desarrollo de innovaciones en cualquier empresa, especialmente en las Pymes, por ser empresas cuya comunicación informal es constante, los trabajadores cuentan con un alto grado de autonomía para realizar sus funciones y existe un alto grado de compromiso entre los trabajadores y la empresa, que facilita la participación de éstos en el desarrollo de las actividades de la Pyme y sobre todo de las innovaciones.

Para seleccionar una empresa de consultoría los empresarios toman en cuenta algunos criterios como por ejemplo el conocimiento del consultor sobre el tema de intervención, la claridad de los alcances, la metodología, la personalización del servicio, y a pesar de ser una manera informal también toman en cuenta la recomendación personal (Zapata et al., 2010).

Las empresas consultoras en materia de recursos humanos, brindan asesoría en las siguientes ramas:

- Asesoría y consultoría preventiva: Servicios que abarcan la implementación de los sistemas de organización, la descripción y va- 
loración de los puestos de trabajo, la evaluación del desempeño, el análisis de los planes de compensación y retribución, el mejoramiento del clima laboral y los procesos de comunicación interna.

- Gestión: Al tener que defender los intereses del cliente frente a los actos de la administración de gestión de recursos humanos.

- Ejecución y representación: Se participa en conjunto con el cliente, o en nombre de él, en la ejecución de sus informes, reportes, asuntos o trámites.

- Asesoría: En el proceso de divulgación para reducir su impacto, es una forma de evitar resistencias, conflictos o deterioro del clima organizacional.

- Capacitación: Es parte fundamental de los servicios de asesoría, está puede ser genérica, ofrecerse en forma abierta y masiva, ya sea por materia o por usuario.

Las Pymes para contratar el servicio de una consultoría deben incurrir en un sacrificio importante del presupuesto, sin embargo, este desembolso representa una inversión, considerando que si no se diagnostica y resuelve el problema, el costo de una mala gestión en recursos humanos puede ser muy perjudicial pues afecta el desempeño de la organización.

El objetivo de este estudio es insertar en el mercado una empresa que brinde los servicios de consultoría, a un precio accesible para las Pymes y que se oferte mediante la red de Internet, de ahí la importancia de conocer acerca de esta nueva forma de hacer negocios.

La Internet se ha extendido en el mundo occidental, son cada vez más personas que acceden a la Red mediante diversos dispositivos como lo son las computadoras portátiles, los teléfonos móviles, las tabletas, lo cual les permite acceder a la información en cualquier momento y lugar; transformación que se inició desde finales del siglo pasado, incidiendo en la forma en que las personas se comunican e intercambian información y por ende en la forma de hacer negocios a nivel mundial (Díaz-Noci, 2010).

Las empresas deben estar preparadas para estos cambios, el personal de manera individual, los grupos y equipos de trabajo, y las estructuras organizativas se han ido modificando hacia una mayor integración organizativa.

La forma en que las personas se relacionan con la información digital está dando un salto cualitativo, de acuerdo con Díaz-Noci (2010) un ejemplo de esto se refleja en el consumo de la música, que ha ido abandonando el soporte físico por usar archivos disponibles en Internet; es decir, de "la cultura de propiedad a la cultura del acceso", a este mercado se orientan las tabletas tipo iPad o la conexión wi-fi, que permiten un recurso ubicuo y gratuito (Díaz-Noci, 2010 ).

Las empresas a nivel mundial han optado por este medio de comercialización y difusión, en la red se encuentran software de administración útiles para el mejoramiento de la gestión, la capacitación en línea en áreas de recursos humanos, listas de proveedores, catálogos de artículos, en fin herramientas como la web $2.0 \mathrm{y}$ las redes sociales están contribuyendo a que las Pymes tengan acceso a información oportuna.

\section{METODOLOGÍA}

El objetivo general de esta investigación consiste en insertar en el mercado nacional los servicios de consultoría en recursos humanos para las Pymes por medio de Internet.

Para analizar la viabilidad de este emprendimiento se plantearon los siguientes objetivos específicos: 
1. Determinar si los directores, gerentes y propietarios de las empresas están dispuestos a contratar los servicios de una empresa consultora en recursos humanos, que sea innovadora en la forma de ofertar el servicio.

2. Reconocer las competencias personales, técnicas, tecnológicas, de influencia, y cognitivas que deben poseer los profesionales que ofrecen servicios de consultoría en las empresas.

3. Investigar las áreas de la administración de recursos humanos en las cuales es primordial el aporte brindado por una empresa de consultoría.

4. Identificar las habilidades tecnológicas requeridas para insertar este emprendimiento en el mercado nacional.

\section{Hipótesis}

Todas las empresas muestran debilidades en diversas áreas, la gestión de recursos humanos es una de las cuales puede presentar deficiencias por lo que se requiere localizar mecanismos compensadores para crear procesos de mejora, en estos casos las empresas de consultoría son una alternativa.

Para las Pymes el contratar los servicios de una consultoría, cuyo costo ronda alrededor los \$ 1500 mensuales, no es fácil debido a las restricciones presupuestarias que poseen, el nivel de ventas no es muy alto y el mercado al que se dirigen es local o muy pequeño, por lo que una empresa de consultoría que ofrezca opciones innovadoras mediante el uso de herramientas tecnológicas que los pequeños y medianos empresarios puedan acceder mediante Internet se convierte en una buena alternativa.

La sociedad en general ha adoptado como parte de su cotidianidad la red de Internet, las pá- ginas web y las redes sociales, como una forma de vida moderna en la cual se llevan a cabo intercambios comerciales, por lo que un emprendimiento novedoso en consultorías de recursos humanos sería ofrecer estos servicios a través de este medio, para llegar de manera oportuna a un mercado de mayor cantidad de usuarios.

A partir del razonamiento anterior es que surgió el planteamiento de las siguientes hipótesis.

- Hipótesis 1: Las Pymes requieren asesoría profesional, pero su presupuesto es limitado.

- Hipótesis 2: Las Pymes utilizan la red de Internet como un medio de comunicación permanente que les proporciona información pertinente para sus negocios, en el cual consultan catálogos, proveedores, realizan transferencias bancarias, entre otras actividades, por lo tanto, los servicios de consultoría deben utilizarla para ser contactados.

Para lograr los objetivos propuestos, la investigación se dividió en un estudio exploratorio. Como un primer acercamiento al tema elegido se realizó una exhaustiva revisión bibliográfica de la teoría relacionada con los temas sobre emprendimientos, empresa, recursos humanos, tecnología de la información, consultorías; además de mantener conversaciones con expertos en la materia para conocer el criterio de ellos al respecto.

Todo lo anterior sirvió de base de conocimiento para el diseño de la encuesta que permitió la recopilación de la información pertinente para comprobar las hipótesis planteadas en la investigación.

A partir de las diferentes teorías consultadas se definieron las competencias de un profesional en recursos humanos que brinde asesoría a los pequeños y medianos empresarios, acorde con las necesidades de los mismos.

Se envió la encuesta por correo electrónico a unas 200 personas, también se entregó el cuestionario personalmente a otros profesionales en 
el área y se obtuvo respuesta de 51, lo cual permitió obtener información para validar la propuesta.

Seguidamente, se organizaron los datos obtenidos para su análisis y la correlación de las variables.

Las principales limitaciones en esta investigación fueron:

1. En relación con el perfil de los encuestados que respondieron la encuesta, en su mayoría son del sector público y laboran en empresas grandes, lo cual provoca respuestas sesgadas.

2. En la encuesta no se incluyó una pregunta relacionada con las competencias digitales, lo cual hubiese enriquecido más el análisis, pues en la actualidad existe una gran gama de opciones a las cuales los empresarios pueden recurrir mediante la web.

En cuanto a los alcances:

1. Se logró obtener información para dar respuesta los objetivos propuestos y a la pregunta que fundamenta este trabajo.

2. Se validaron las hipótesis planteadas.

\section{RESULTADOS}

Las personas que respondieron la encuesta son profesionales en administración con diversos énfasis, la mayoría en recursos humanos, se contó además con el aporte de otros especialistas en finanzas, mercadeo, producción, gerencia, administración pública, que trabajan en empresas públicas y privadas.

El único dato demográfico que se consultó es el de la edad, esta variable es de utilidad como una referencia general, en tanto aportó a la investigación un dato relevante que se correlaciona con las otras variables estudiadas (ver figura 1).
FIGURA 1

\section{EDADES DE LOS ENTREVISTADOS}

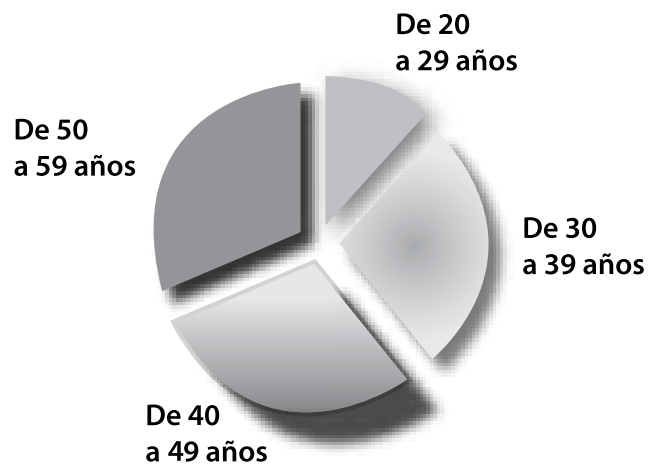

Fuente: elaboración propia

Un alto porcentaje de los encuestados trabajan en el sector público en puestos de dirección, gerencia o jefatura y solo 10\% labora para el sector privado, lo que responde a dos factores, el primero fue que el cuestionario se envió a los contactos de la investigadora quien labora en el sector público y el segundo es que la muestra es de profesionales en administración y el Estado es el más importante empleador de profesionales en Costa Rica.

\section{FIGURA 2}

\section{CONTRATACIÓN DE UNA CONSULTORÍA}

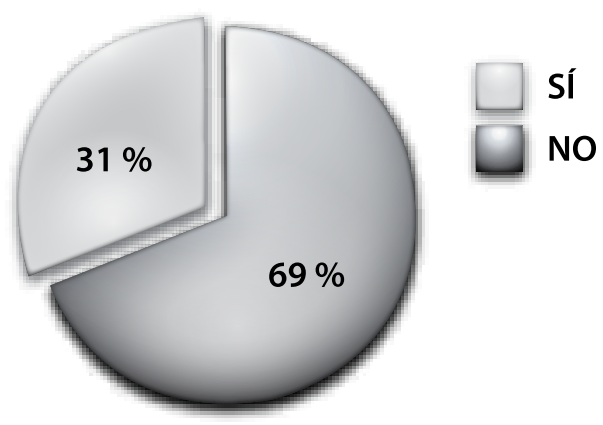

Fuente: elaboración propia. 
El objetivo principal de la investigación consistió en analizar la viabilidad de insertar en el mercado una empresa de consultoría, por lo que se formuló la pregunta para conocer si las empresas contratarían los servicios de una consultoría, la respuesta de la mayoría fue positiva, lo cual indica que la demanda existe, y los directores y gerentes están dispuestos a proponer en la empresa esta posibilidad como una opción para resolver problemas internos en materia de recursos humanos.

Existe un porcentaje que corresponde a la tercera parte de la muestra que respondió no considerar la contratación de una empresa consultora. En la tabla 2 se resume las razones del porqué sí contratarían una empresa consultora en recursos humanos y del porqué no lo harían, de acuerdo con la información aportada por los encuestados en sus respuestas.
A la pregunta ¿qué medio utilizaría para contactar una empresa de consultoría?, casi la mitad de los encuestados eligieron como primera opción referencias personales, es decir, por medio de otras personas a quienes les han brindado servicio, el segundo medio de contacto sería la red de Internet, donde se incluyen páginas web y redes sociales, lo que fundamenta este emprendimiento, ya que el principal medio de promoción del mismo es mediante la red de Internet (Figura 3).

Cerca de $60 \%$ de quienes respondieron la encuesta son profesionales con edades entre los 41 y 60 años, por lo que es probable que el medio de contacto que prefieran no sean las herramientas tecnologías digitales a las cuales se tiene acceso por medio de la red de Internet.

TABLA 2

\section{RAZONES QUE JUSTIFICAN 0 IMPIDEN LA CONTRATACIÓN DE UNA CONSULTORÍA SEGÚN LOS ENCUESTADOS}

\begin{tabular}{|c|c|}
\hline No la contrataría & Sí la contraría \\
\hline $\begin{array}{l}\text { - } \quad \text { La empresa tiene la capacidad para resolver los asuntos en esta } \\
\text { materia sin asesoría externa. } \\
\text { - } \quad \text { En la empresa se han contratado y no han aportado nada nuevo. } \\
\text { - } \quad \text { Por falta de presupuesto. } \\
\text { - } \quad \text { No, porque la empresa cuenta con un equipo interdisciplinario y } \\
\text { capacitado. } \\
\text { - } \quad \text { Por aspectos legales no es permitido contratar servicios externos. } \\
\text { - No, cuando la cantidad de empleados es pequeña. }\end{array}$ & $\begin{array}{l}\text { - } \quad \text { En caso de que el problema se salga del conocimiento y especialidad, } \\
\text { del dominio interno. } \\
\text { - } \quad \text { Permite una visión más objetiva. } \\
\text { - } \quad \text { Resolver de manera oportuna. } \\
\text { - } \quad \text { Por las limitaciones cognitivas de los involucrados internamente. } \\
\text { - } \quad \text { Por el criterio profesional externo. } \\
\text { - } \quad \text { Para eliminar sesgos. } \\
\text { - } \quad \text { Se aprovechan nuevas tendencias en la materia. } \\
\text { - } \quad \text { Evita influencias de los criterios internos. } \\
\text { - } \quad \text { Dan resultados positivos. }\end{array}$ \\
\hline
\end{tabular}

Fuente: elaboración propia. 
Figura 3

\section{MEDIO DE CONTACTO PARA EMPRESA CONSULTORA}

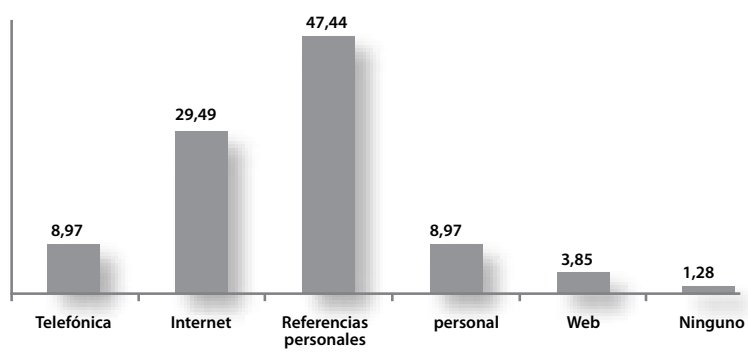

Fuente: elaboración propia.

Para los jóvenes es usual buscar opciones de compra de bienes y servicios, por medio de Internet, incluso las empresas se promocionan en las populares redes sociales como Facebook, mientras que por hábitos y costumbres adoptadas desde décadas anteriores, las personas de mayor edad tienden a recurrir a medios de contacto tradicionales. El emprendimiento propuesto en esta investigación utilizaría las redes sociales como uno de los medios de promoción.

Para emprender un negocio se deben poseer habilidades, destrezas y conocimientos que favorezcan la probabilidad de éxito, por lo que en la encuesta se indagó acerca de las competencias personales, técnicas, tecnológicas, de influencia y cognitivas que deben dominar los profesionales que ofrecen servicios de consultoría en las empresas.

Los resultados obtenidos ante el cuestionamiento citado aparecen reflejados en la Figura 4.

La competencia personal a la que mayor relevancia le asignaron los especialistas en administración encuestados es la experiencia en recursos humanos, seguida por la formación académica, dos aspectos cruciales en la prestación de servicios en consultoría.
FIGURA 4

\section{COMPETENCIAS PERSONALES}

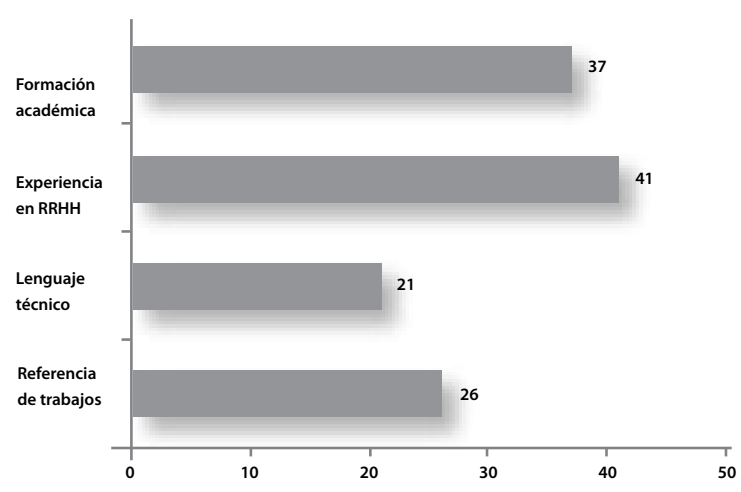

Fuente: elaboración propia.

Por otra parte, el conocimiento profesional de la gestión de recursos humanos y la legislación vigente en materia laboral son las competencias cognitivas que los encuestados colocaron en primer lugar de importancia, 80\% valoraron ambas áreas como muy importantes, en las que existe una necesidad de capacitación por parte de los propietarios y administradores de las Pymes, debido a que en su mayoría no son profesionales en esta materia.

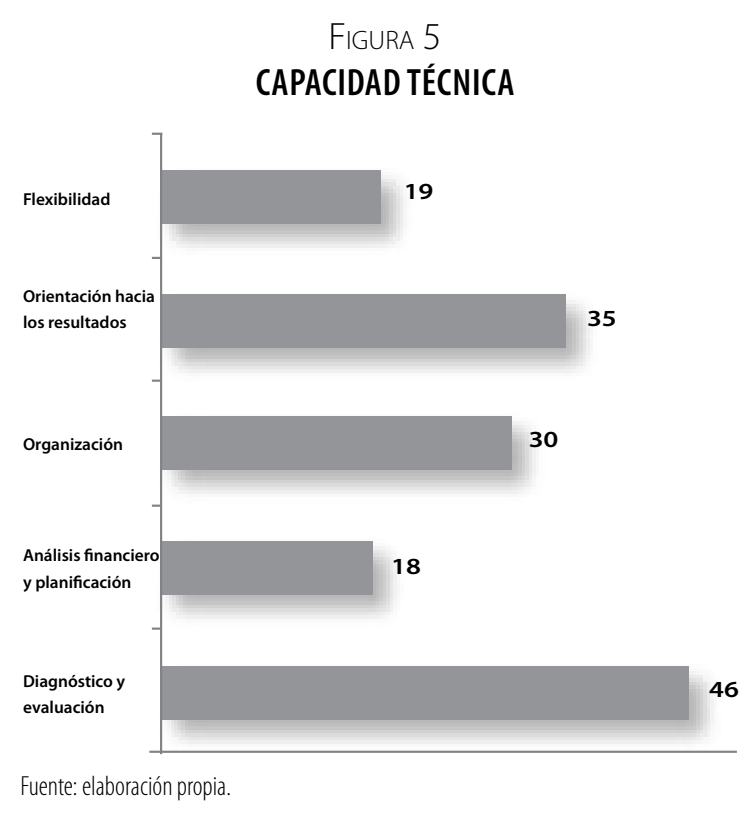


Para los encuestados la capacidad técnica de mayor importancia al contratar una consultorías fue la capacidad de diagnóstico y evaluación, capacidad vinculada a la tarea fundamental de un consultor que consiste en identificar e investigar los problemas relacionados con la gestión empresarial, él debe elaborar un diagnóstico preciso que le permita recomendar las medidas apropiadas para resolver las situaciones adversas (ver figura 5).

La otra capacidad técnica a la que le otorgaron gran importancia fue la orientación hacia los resultados, de hecho, toda empresa busca ser exitosa en el mercado y para lograrlo debe implementar estrategias que le permitan alcanzar las metas formuladas, por lo que un consultor debe tener claro la orientación de la empresa y direccionar sus propuestas hacia el logro de esos resultados.

El tercer objetivo específico de esta investigación consistió en identificar las habilidades tecnológicas que se requieren para insertar este emprendimiento en el mercado nacional. Por lo que se propuso conocer las competencias tecnológicas que para los encuestados deben ser relevantes para una empresa de consultoría, en otras palabras, qué esperan los posibles clientes de los profesionales que brindan asesoría en el uso de herramientas tecnológicas. Es decir, herramientas que le permiten a las organizaciones generar, acceder, almacenar y transferir el conocimiento existente en la empresa.

De acuerdo con las respuestas obtenidas la competencia tecnológica más importante es el uso de software de sistemas gerenciales, sin embargo, en promedio un tercio de los informantes señaló como muy importante el uso de páginas web y de las redes sociales, lo cual marca una tendencia interesante al relacionarla con la variable de medios de contacto, donde un promedio de 30\% dice que utilizaría Internet como medio para contactar el servicio de una empresa consultora.

\section{FIGURA 6 \\ COMPETENCIAS TECNOLÓGICAS}

Porcentaje

Muy importante

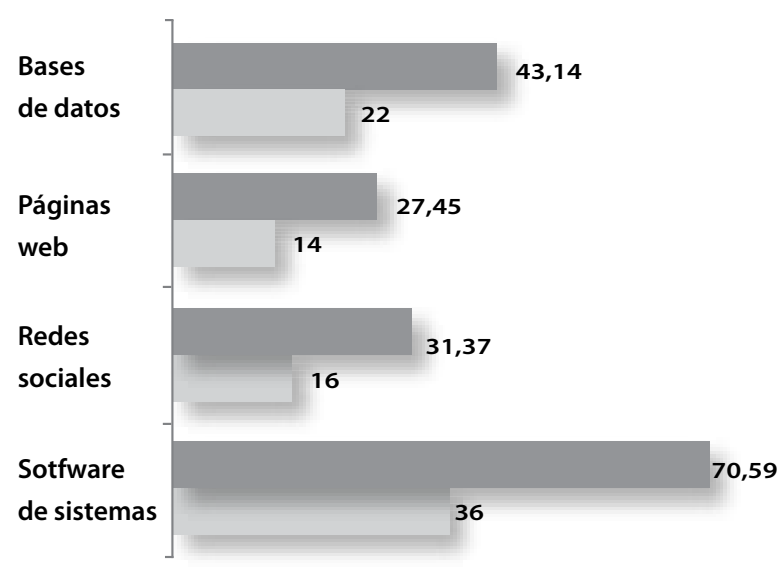

Fuente: elaboración propia.

En este caso es necesario tomar en cuenta que la mayoría de quienes respondieron la encuesta superan los 41 años (60\%), mientras entre los 21 y 30 solo 12\%, lo cual podría estar relacionado con la habilidad y disposición del uso de Internet, consultas a páginas web y la participación activa en las redes sociales, tanto en el modo de contactar un servicio como en la importancia que le asignan a esta competencia.

Las competencias de influencia, todas las que fueron incluidas en el cuestionario, representan de gran importancia para los profesionales entrevistados, siendo la comunicación efectiva y el trabajo en equipo los indicadores que ocupan el primer lugar, pero, no se puede decir que los otros indicadores propuestos no sean muy relevantes tal como la orientación hacia el cliente interno, la motivación, el aprendizaje continuo y la gestión de las personas.

En la encuesta se consultó a los profesionales si ellos estarían dispuestos a contratar una empresa consultora innovadora que ofrezca los servicios aprovechando las redes sociales y las 
páginas web, la respuesta de 70\% fue positiva, además se les silicito que anotaran lo que esperaban de la empresa. En resumen ellos citaron los siguientes aspectos:

1. Asesoramiento en las áreas de recursos humanos

2. Servicio actualizado y personal capacitado

3. Objetividad y ética

4. Ideas innovadoras generadoras de un valor agregado

5. Efectividad de los resultados

6. Informes parciales debidamente fundamentados

7. Casos documentados de éxito

\section{FIGURA 7 \\ PERCEPCIÓN SOBRE QUÉ ES UNA CONSULTORÍA}

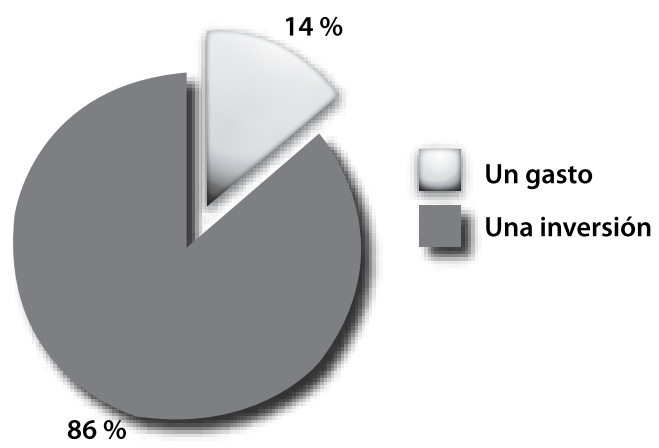

Fuente: Elaboración propia.

Otro aspecto que se analizó en esta investigación es si consideraban la contratación de una consultoría un gasto o una inversión, esto porque los pequeños y medianos empresarios disponen de un presupuesto muy limitado y el pagar por este servicio es un costo que deben planificar, la idea es proponerles el servicio a un precio accesible y del que recibirán beneficios en tanto la implementación de las soluciones recomendadas se traduzca en mejoramiento de la gestión.

Para la gran mayoría de los encuestados la contratación de una consultoría es una inversión por diversas razones, entre los que citaron:

1. Ayuda con la mejora de la gestión,

2. permite el desarrollo en la función de recursos humanos,

3. produce soluciones efectivas a la empresa,

4. proporciona motivación a los empleados,

5. incrementa la productividad,

6. aporta visión externa a la empresa y

7. favorece el intercambio de conocimiento e información.

Los que consideran la consultoría como un gasto argumentaron lo siguiente:

1. Los problemas se deben resolver a nivel interno para lo cual la empresa dispone de expertos,

2. los beneficios de la propuesta elaborada por un consultoría son a largo plazo,

3. para las empresas pequeñas con poco personal es un gasto innecesario ya que no siempre se pueden implementar las propuestas.

Estas argumentaciones en contra podrían estar influenciadas por las características laborales de los profesionales que respondieron el cuestionario, la mayoría de ellos trabajan para el sector público, cuentan así con departamentos de 
recursos humanos y son grandes organizaciones con más de 100 empleados. Sin embargo, las empresas de consultoría ofrecen servicios a todo tipo de empresa sea grande, mediana o pequeña y no siempre se dispone de expertos en recursos humanos en las organizaciones.

En general los encuestados coincidieron en que las empresas consultoras deben dar garantía del trabajo realizado, y en que la implementación de la propuesta y las recomendaciones de la consultoría quedarían en manos de la gerencia. Sin embargo, desde la perspectiva de investigadores sobre el tema de consultorías como Zapata (2010) el servicio debe ser prestado por personal calificado, que contribuya a la solución de los problemas, y que en la tercera etapa de la consultoría donde se evalúan los resultados obtenidos, el consultor acompañará al cliente a implementar los ajustes requeridos; en otras palabras, la consultoría no termina en el diseño y entrega de la propuesta, el consultor debe acompañar al cliente en todo el proceso, incluyendo la implementación, con el propósito último de mejorar la gestión.

Para complementar el estudio y conocer cuáles son las áreas en que las empresas requieren asesoría en materia de recursos humanos se preguntó acerca del grado de importancia por área, por lo que se utilizó un ítem de selección múltiple (ver figura 8)

Los encuestados marcaron como áreas de mayor importancia la evaluación del desempeño y la capacitación y desarrollo, seguido por el análisis de puestos y el reclutamiento y selección. Por último, se señalaron las compensaciones y la legislación laboral, las cuales fueron consideradas dentro del nivel de muy importante por un tercio de los encuestados, a pesar de que el conocimiento en materia de legislación laboral ocupó $80 \%$ en grado de importancia cuando se valoraron las competencias cognitivas, lo cual reflejó una necesidad de capacitación por parte de las Pymes en la materia.

\section{FIGURA 8 \\ GRADO DE IMPORTANCIA POR ÁREA DE RRHH (ÍTEM DE SELECCIÓN MÚLTIPLE)}

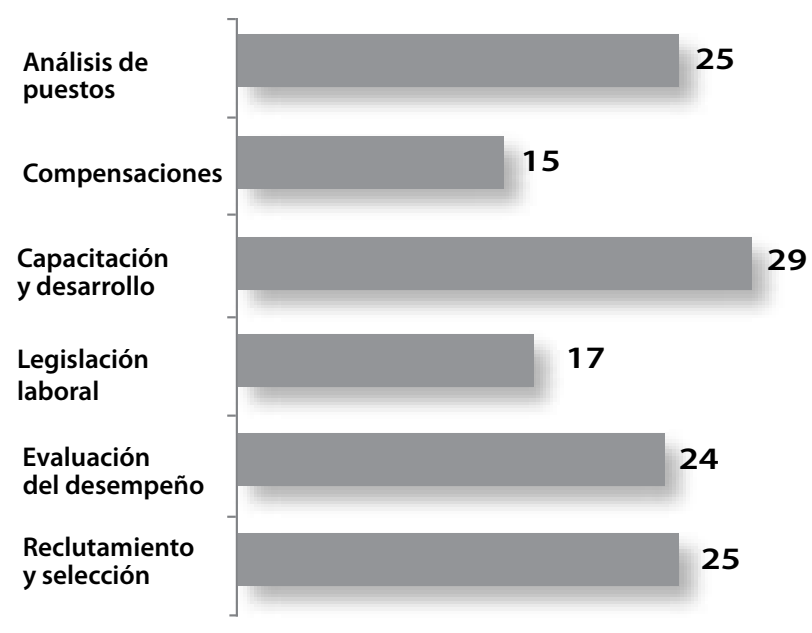

Fuente: elaboración propia.

Finalmente se le consultó a los encuestados acerca de las áreas específicas que no fueron contempladas en este cuestionario, aquellas en las que las empresas requieren asesoría en materia de recursos humanos:

- Análisis del clima organizacional y la cultura

- Alineamiento del recursos humano a la estructura

- Relaciones laborales y solución de conflictos

- Salud e higiene ocupacional

- Clasificación y valoración de puestos

- Inteligencia emocional

- Capacitación en software como herramienta para agilizar procesos 


\section{CONCLUSIONES}

Para insertar este emprendimiento es necesario considerar que la capacidad económica de las Pymes es limitada, lo cual condiciona la posibilidad de contratar el servicio de una empresa consultora, a pesar de las dificultades en momentos de crisis, se debe valorar esta opción como un camino para el mejoramiento en la gestión así como para alcanzar las metas establecidas, ya que un proceso de consultoría contribuye al planteamiento de una estrategia competitiva, direccionada a captar el mercado e identificar oportunidades de negocio mediante el servicio de personal capacitado.

Los empresarios son conscientes de la importancia y la necesidad de contar con el asesoramiento que les puede brindar una empresa de consultoría en recursos humanos, por lo que este emprendimiento debe insertarse mediante los diversos medios de contacto al cual tienen acceso los pequeños y medianos empresarios, innovando en la forma de ofertar el servicio pues está comprobado que los dueños de las Pymes realizan diversas transacciones mediante la red de Internet y el comercio electrónico; es decir herramientas que se constituyen en una nueva forma de hacer negocios.

Para los gerentes y directores de las empresas las competencias más importantes que deben poseer los profesionales en servicios de consultoría son la experiencia en recursos humanos, el conocimiento de la gestión de recursos humanos y el conocimiento en materia de legislación laboral; además la competencia técnica más que sobresale es la capacidad para diagnosticar y evaluar problemas que afectan la empresa para así poder plantear soluciones viables.

A las competencias tecnológica que mayor importancia les asignaron los encuestados fueron el uso de software de administración -entre ellos software de gestión, de facturación y stock para Pymes-, de fácil acceso mediante la red, y a las consultas de las bases de datos; no obstante, no se puede despreciar el que las redes sociales y las páginas web también son un excelente medio de promoción.

Las áreas de la administración de recursos humanos en las cuales los gerentes consideran de vital importancia el aporte profesional de una consultoría son:

1. Evaluación del desempeño

\section{Capacitación y desarrollo}

\section{Legislación laboral}

Es evidente que este emprendimiento es viable ya que las empresas requieren asesoría en la materia y que los gerentes y propietarios estarían en la disposición de contratar los servicios de una empresa de consultoría innovadora.

Las herramientas tecnológicas que se proponen para insertar en el mercado nacional este emprendimiento son las redes sociales, como medio de promoción, así como el diseño y la administración de una página web de fácil acceso que contenga toda la información pertinente y actualizada.

\section{RECOMENDACIONES}

La empresa de consultoría deberá ofrecer el servicio según las necesidades de cada cliente y adecuarse a la cultura y los valores organizacionales.

Al momento de considerar los precios por los servicios de consultoría, se debe tomar en cuenta el mercado meta, por lo que se debe ofrecer flexibilidad en el precio y en los esquemas de forma de pago, lo que definitivamente se convertirá en una ventaja para la consultora. 
Los profesionales que ofrecerán los servicios en esta consultora ostentarán formación académica en recursos humanos y experiencia comprobada en el área.

Independientemente del puesto, se les capacitará en herramientas tecnológicas digitales a quienes trabajen en la consultora, con el fin de que estén actualizadas y brinden un servicio de calidad.

Se propone además como futuras líneas de investigación:

a) Diseñar una propuesta de asesorías en recursos humanos para los pequeños y medianos empresarios.

b) Conocer las competencias que deben reunir los profesionales que les ofrecen servicios de consultorías a las Pymes.

\section{Referencias}

Alonso, E., Lebendiker, M., Petry, P. y Zevallos, E. (2010). Diagnóstico sobre la situación del Emprendedurismo. Cartago:Asociación Incubadora Parque TEC.

Barley, S. y Kunda, G. (1992). Design and devotion: surges of rational and normative ideologies of control in management discourse. Administrative Science Quarterly, 37(3), 363-399.

Baumol, W.J. (1997). Pareto Optimal Sizes of Innovation Spillovers, Research Report \# 97-42. The C.V. Starr Center for Applied Economics, Department of Economics, New York University.

Brockhaus, R. H. (1980). Risk taking propensity of intrepreneurs. Academy of Management Journal. 23(3), 509-520.

Chiavenato, I. (2007). Gestión del talento humano. Bogotá, Colombia:McGraw-Hill.

Díaz-Noci, J. (2010). Medios de comunicación en Internet: algunas tendencias. El profesional de la información, 19 (6), 561-567.

Gilder, G. (1984) The spirit of enterprise. New York: Simon and Schuster.
Gómez, D. y Múnera, J.I. (1998). El mundo de la consultoría empresarial. Tecnología Administrativa, 12(28), 13-39.

González, M. (2001). El papel de la Internet en los sistemas de información empresariales. Informations Science \& Technology. Recuperado de: http://www.ist.caltech.edu/

Hisrich, R., Peters, M. y Shepherd, D. (2005). Entrepreneurship. Madrid: MacGraw Hill.

Jennings, D. (1994). Multiple Perspectives of Entrepreneurship. USA: South-Western Pub.

Kantis, H. (2004) Desarrollo emprendedor: América Latina y la experiencia internacional. Banco Interamericano de Desarrollo. Recuperado de: http://www.fundes.org/ uploaded/content/publicacione/1572456652.pdf

Kubr, M. (2002). La consultoría de empresas. Guía de la profesión (3ra ed.). México DF: Limusa.

McClelland, D. C. (1961). The achieving society. New York: The Free Press.

Observatorio de Mipymes. (2008). Hacia el estado de las Mipymes: Primer diagnóstico nacional de Mipymes. Costa Rica: UNED.

Roberts, D. y Woods, C. (2005). Changing the world on a shoestring: The concept of social entrepreneurship. University of Auckland Business Review, 7(1), 45-51.

Rubio, A. y Aragón, A. (2006). Competitividad y recursos estratégicos en las Pymes. Revista de Empresa. №17 Julio - Septiembre, 32- 49.

Schuler, R.S. y Jackson, S. (1987). Linking competitive strategies with human resources management practices. The Academy of Management Executive, 1(3), 207- 219.

Schumpeter, J. A. (1934). The Theory of Economic Development. Cambridge: Harvard University Press.

Thompson, I. (2010). Concepto de empresa. Recuperado de: http://www.crecenegocios.com/concepto-deempresario

Ulrich, D. (1997). Human Resource Champions: The Next Agenda for Adding Value and Delivering Results. Boston, MA: Harvard Business Press.

Zapata, E., Rivillas, C. y Cardona, H. (2010). LA CONSULTORÍA DE GESTIÓN HUMANA EN EMPRESAS Medianas. (Español). Estudios Gerenciales , 26 (114), 149-168.

Recibido: 14 de noviembre de 2011 Aceptado: 10 de diciembre de 2012 